\title{
Resonance in-plane magnetic field effect as a means to reveal the Fulde-Ferrell-Larkin-Ovchinnikov state in layered superconductors
}

\author{
M. D. Croitoru and A. I. Buzdin \\ Université Bordeaux I, LOMA, UMR 5798, F-33400 Talence, France \\ (Received 15 April 2012; revised manuscript received 28 May 2012; published 7 August 2012)
}

\begin{abstract}
The vector potential of a parallel magnetic field produces a modulation of the interlayer coupling between adjacent superconducting layers. In some cases the period of this modulation can coincide with the period of the Fulde-Ferrell-Larkin-Ovchinnikov (FFLO) modulation of the superconducting order parameter. Such a resonance condition results in cusps on the temperature and in-plane angular dependencies of the upper critical field $H_{c 2}$. This effect can open up a possibility to unambiguously evidence a spatially modulated superconducting phase in layered conductors. Remarkably, the proposed signature of the FFLO state is directly based on the main feature of the FFLO state, the spatial modulations of the order parameter.
\end{abstract}

DOI: 10.1103/PhysRevB.86.064507

PACS number(s): 74.70.Kn, 74.78.Fk, 74.25.Op

\section{INTRODUCTION}

The effect of high magnetic fields on the superconducting state in quasi-one- and two-dimensional electronic structures has been a subject of increasing interest during the last several decades. ${ }^{1,2}$ This has been motivated, in particular, by the experimental observation of a field-dependent upturn of the superconductivity onset curve at low temperatures in the charge-transfer salts based on the bis(ethylenedithio)tetrathiafulvalene (BEDTTTF) and on the tetramethyltetraselenafulvalene (TMTSF; Bechgaard salts) molecules, which exhibit highly anisotropic layered structures and hence have features of systems with reduced dimensionality. ${ }^{3-5}$ The Fulde-Ferrell-LarkinOvchinnikov (FFLO) ${ }^{6,7}$ state with a modulated superconducting order parameter could be at the origin of the observed effect.

The layered organic superconductors are very suitable for the FFLO observation. Indeed, for an in-plane magnetic field the orbital suppression of superconducting correlations is reduced and for singlet-paired superconductivity the Zeeman response dominates the orbital response at low temperatures. Therefore the superconducting pairing is mainly controlled by the Pauli paramagnetic limit, at which the energy difference of the oppositely directed spins becomes comparable to the condensation energy. The known layered organic superconductors are in the clean limit, $\xi_{0}=\hbar v_{F} / \pi \Delta_{0}>l,{ }^{8}$ where $l$ is the mean free path and $\Delta_{0}$ is the superconducting energy gap at $T=0$ and $H=0$, which is another necessary condition to observe the modulated superconducting phase since it is very sensitive to the presence of nonmagnetic impurities. ${ }^{9,10}$ Furthermore, strong anisotropy of organic layered compounds resulting in the increase of nesting topology of their Fermi surfaces also favors the FFLO state. ${ }^{11}$

A possible FFLO state formation has been evidenced in the experiments with layered organic superconductors,,${ }^{14-14}$ when an external magnetic field is aligned along the conducting planes. The anomaly in the thermal conductivity for the clean organic sample $\lambda$-(BETS) ${ }_{2} \mathrm{GaCl}_{4},{ }^{15}$ the calorimetric and magnetic torque evidence for the appearance of an additional first-order phase transition line within the superconducting phase in the in-plane high field regime for organic sample
$\kappa$-(BEDT-TTF $)_{2} \mathrm{Cu}(\mathrm{NCS})_{2},{ }^{3,4}$ as well as an anomalous inplane anisotropy of the onset of superconductivity in a (TMTSF) ${ }_{2} \mathrm{ClO}_{4}$ conductor ${ }^{5,8}$ have been interpreted as being related to a stabilization of the superconducting phase with the modulated order parameter in real space. The FFLO state is expected to exist also in heavy fermion superconductors. ${ }^{16-19}$

Previously it has been demonstrated that in the tilted magnetic field the interplay between the orbital and the spin effects gives rise to the very peculiar angular upper critical field behavior. ${ }^{20-23}$ In the case of the parallel orientation of the magnetic field, the orbital effect, though small, provides an interesting possibility to sample the direction of the FFLO modulation-the in-plane anisotropy of the onset of superconductivity should change dramatically in the FFLO state. ${ }^{24}$ The vector potential of the parallel magnetic field results in a modulation of the interlayer coupling with the period $\lambda_{H}=2 \phi_{0} / d H$, where $d$ is the interlayer distance and $\phi_{0}=\pi c / e$. The period of this modulation may interfere with the in-plane FFLO modulation leading to the anomalies in the critical field behavior. The strongest effect occurs when the magnetic length $\lambda_{H}$ coincides, i.e., in the resonance with the period of FFLO modulation $\lambda_{\text {FFLO }}$ (for $T=0$, $\left.\lambda_{\mathrm{FFLO}}^{0}=\pi \hbar v_{F} / \Delta_{0}=\pi^{2} \xi_{0}\right)$. The upper critical field at $T=0$ observed in $\kappa$-(BEDT-TTF $)_{2} \mathrm{Cu}(\mathrm{NCS})_{2}$ is $H_{c 2} \simeq 30 \mathrm{~T}$. Since $d=1.62 \mathrm{~nm}^{25} \xi_{0}=7-9 \mathrm{~nm}$ this leads to $\lambda_{H}=85 \mathrm{~nm}$ and $\lambda_{\mathrm{FFLO}}^{0}=70-90 \mathrm{~nm}$. In $(\mathrm{TMTSF})_{2} \mathrm{ClO}_{4}, H_{c 2}(0)=5 \mathrm{~T}$, $d=1.31 \mathrm{~nm}, \xi_{0}=45 \mathrm{~nm}$ resulting in $\lambda_{H}=630 \mathrm{~nm}$, and $\lambda_{\mathrm{FFLO}}^{0}=444 \mathrm{~nm}$. In $\lambda$-(BETS $)_{2} \mathrm{FeO}_{4}$ the lower $H_{c 2}(0)=18 \mathrm{~T}$, $d=1.85 \mathrm{~nm}, \xi_{0}=8.5 \mathrm{~nm}$ resulting in $\lambda_{H}=130 \mathrm{~nm}$, and $\lambda_{\mathrm{FFLO}}^{0}=85 \mathrm{~nm}$. For these compounds $\lambda_{\mathrm{FFLO}}^{0}<\lambda_{H}^{0}$ at $T=0$. The FFLO modulation appears only at $T<T^{*} \simeq 0.56 T_{c 0},{ }^{26}$ with a wave vector $\mathbf{q}$ growing from $q=0$ to $q_{0}=q(T=$ $0)=2 \Delta_{0} / \hbar v_{F}$ with decreasing temperature. Therefore if the condition $\lambda_{\text {FFL }}^{0}<\lambda_{H}^{0}$ is satisfied at $T=0$, then at some finite temperature $T$ the resonance condition $\lambda_{\mathrm{FFLO}}(T)=\lambda_{H}(T)$ should be realized. This corresponds to the situation when the strongly overlapping Josephson vortices form a rectangular lattice with its centers just above the nodes of the order parameter. Note that previously such mechanism of pinning of Josephson vortices by the nodes of FFLO modulation was suggested in Ref. 27 and observed in organic superconductor $\lambda$-(BETS $)_{2} \mathrm{FeCl}_{4}{ }^{28}$ The possibility of a resonance between 
FFLO and interlayer coupling was not considered in Ref. 24 and the analysis of this regime is the main subject of the present paper. At the resonance the orbital effect is strongly enhanced resulting in a cusplike change of the $H_{c 2}(T)$ dependence. The experimental observation of these anomalies can be a strong signature of the FFLO phase formation in a layered superconducting sample. The proposed tool for exhibiting the FFLO phase is directly based on its main feature, the spatial modulations of the order parameter.

\section{THEORETICAL MODEL}

We consider a system consisting of layers with good conductivity in an $x y$ plane stacked along the $z$ axis. The single-electron spectrum is taken as follows:

$$
E_{\mathbf{p}}=\frac{p_{x}^{2}}{2 m_{x}}+\frac{p_{y}^{2}}{2 m_{y}}+2 t \cos \left(p_{z} d\right) .
$$

As was demonstrated in Ref. 29 the anisotropic effective mass model can be reduced to the isotropic one (IM model) by a scaling transformation and corresponding renormalization of the magnetic field. Therefore in this work we consider only the isotropic spectrum and moreover to neglect the critical fluctuation effects we assume that the coupling between layers is $t \gg T_{c 0}^{2} / E_{F} \cdot{ }^{30}$

For the in-plane magnetic field, with amplitude $H$, applied at angle $\alpha$ with the $x$ axis (the direction of the FFLO modulation vector) we may choose the vector potential along the $z$ axis, with $A_{z}=-x H \sin \alpha+y H \cos \alpha$. Assuming that the vector potential varies slowly at the interlayer distances and taking into account that the system is near the secondorder phase transition, we employ the linearized Eilenberger equation for a layered superconductor in the form ${ }^{31}$

$\left[\Omega_{n}+2 i t \sin \left(p_{z} d\right) \sin (\mathbf{Q} \cdot \mathbf{r})+\frac{1}{2} \mathbf{v}_{F} \cdot \nabla\right] f_{\omega_{n}}\left(\mathbf{n}, \mathbf{r}, p_{z}\right)=\Delta(\mathbf{r})$,

where $\Omega_{n} \equiv\left|\omega_{n}\right|+i h \operatorname{sgn}\left(\omega_{n}\right), h=\mu_{B} H$ is the Zeeman energy, $\mathbf{v}_{F}=v_{F} \mathbf{n}$ is the in-plane Fermi velocity, and the vector $\mathbf{Q}=\left(\pi d H / \phi_{0}\right)[-\sin \alpha, \cos \alpha, 0]$ is perpendicular to the magnetic field. Here $f_{\omega}\left(\mathbf{n}, \mathbf{r}, p_{z}\right)$ is the anomalous quasiclassical Green function integrated over the energy near the Fermi surface. The order parameter is defined self-consistently as $\Delta(\mathbf{r})=\lambda \pi T \sum_{n}\left\langle f_{\omega_{n}}\left(\mathbf{n}, \mathbf{r}, p_{z}\right)\right\rangle$, with $\langle\cdots\rangle$ meaning the average over the Fermi surface.

In two-dimensional (2D) superconductors the FFLO state appears as a modulated order parameter with a wave vector q whose direction is determined by the crystal field effects. ${ }^{32}$ The orientation of the FFLO modulation vector is arbitrary in the pure Pauli-limited regime in the case of an elliptic Fermi surface. The crystal field introduces deviations from the ellipticity and pins the FFLO modulation vector in a certain direction. For example, by considering the simplified model of the square Fermi surface (SM) with constant modulus of Fermi velocity to describe the in-plane electron motion one can see that the directions of the FFLO modulation vector are along the diagonals of the Fermi square. The symmetry of the order parameter can provide an additional source of pinning for the modulation vector. In this work we consider the $s$-wave pairing but we expect that a similar situation occurs in the case of $d$-wave pairing.

To describe the angular dependence of the upper critical field in the FFLO phase in quasi-2D superconductors we have to go beyond the simple exponential solution $f_{\omega_{n}}\left(\mathbf{n}, \mathbf{r}, p_{z}\right) \sim$ $\exp (i \mathbf{q r})$ and incorporate the orbital effects, which add the higher harmonics in FFLO modulation, $\mathbf{q} \pm m \mathbf{Q}$. Therefore, the solution of Eq. (2) can be written as

$$
f_{\omega_{n}}\left(\mathbf{n}, \mathbf{r}, p_{z}\right)=e^{i \mathbf{q} \cdot \mathbf{r}} \sum_{m} e^{i m \mathbf{Q} \cdot \mathbf{r}} f_{m}\left(\omega_{n}, \mathbf{n}, p_{z}\right) .
$$

At the same time only the even harmonics are present in the order parameter $\Delta(\mathbf{r})$,

$$
\Delta(\mathbf{r})=e^{i \mathbf{q} \cdot \mathbf{r}} \sum_{m} e^{i 2 m \mathbf{Q} \cdot \mathbf{r}} \Delta_{2 m} .
$$

We derive a second-order approximation in the small parameter $t / T_{c 0}$ to the solution of Eq. (2). This implies that the higher harmonics in $\Delta(\mathbf{r})$ are small [e.g., $\Delta_{2} \sim\left(t / T_{c 0}\right)^{2} \Delta_{0}$ ] and usually can be neglected. ${ }^{24}$ However one may notice that at condition $\mathbf{q}-2 \mathbf{Q}=-\mathbf{q}$, the harmonics $\Delta_{0}$ and $\Delta_{-2}$ are the same and should be considered on equal footing. This special situation results in the unusual temperature and angular dependence of the critical field and it was not considered in Ref. 24. Substituting Eqs. (3) and (4) into Eq. (2) and retaining the terms up to the second harmonic $\Delta_{ \pm 2}$ one gets the following system of coupled equations:

$$
\begin{gathered}
L_{n}(\mathbf{q}) f_{0}+\tilde{t} f_{-1}-\tilde{t} f_{1}=\Delta_{0}, \\
L_{n}(\mathbf{q} \pm \mathbf{Q}) f_{ \pm 1} \pm \tilde{t} f_{0} \mp \tilde{t} f_{ \pm 2}=0, \\
L_{n}(\mathbf{q} \pm 2 \mathbf{Q}) f_{ \pm 2} \pm \tilde{t} f_{ \pm 1} \mp \tilde{t} f_{ \pm 3}=\Delta_{ \pm 2}, \\
L_{n}(\mathbf{q} \pm 3 \mathbf{Q}) f_{ \pm 3} \pm \tilde{t} f_{ \pm 2}=0,
\end{gathered}
$$

where $f_{m} \equiv f_{m}\left(\omega_{n}, \mathbf{n}, p_{z}\right), L_{n}(\mathbf{q})=\Omega_{n}+i \mathbf{v}_{F} \mathbf{q} / 2$, and $\tilde{t}=$ $t \sin \left(p_{z} d\right)$. From Eqs. (7) and (8) one gets

$$
\left[L_{n}(\mathbf{q} \pm 2 \mathbf{Q})+\frac{t^{2}}{L_{n}(\mathbf{q} \pm 3 \mathbf{Q})}\right] f_{ \pm 2} \pm \widetilde{t} f_{ \pm 1}=\Delta_{ \pm 2} .
$$

Substitution of $f_{ \pm 1}$, obtained from Eq. (6),

$$
f_{ \pm 1}=\mp \frac{\tilde{t} f_{0}}{L_{n}(\mathbf{q} \pm \mathbf{Q})} \pm \frac{\tilde{t} f_{ \pm 2}}{L_{n}(\mathbf{q} \pm \mathbf{Q})},
$$

when taking into account that $f_{0} \approx \Delta_{0} / L_{n}(\mathbf{q})$, gives the equation for the second harmonic of the pair amplitude

$$
\begin{aligned}
& {\left[L_{n}(\mathbf{q} \pm 2 \mathbf{Q})+\frac{\tilde{t}^{2}}{L_{n}(\mathbf{q} \pm 3 \mathbf{Q})}+\frac{\tilde{t}^{2}}{L_{n}(\mathbf{q} \pm \mathbf{Q})}\right] f_{ \pm 2}} \\
& -\frac{\tilde{t}^{2} \Delta_{0}}{L_{n}(\mathbf{q}) L_{n}(\mathbf{q} \pm \mathbf{Q})}=\Delta_{ \pm 2} .
\end{aligned}
$$

Substitution of $f_{ \pm 1}$ from Eq. (10) and $f_{ \pm 2} \approx \Delta_{ \pm 2} / L_{n}(\mathbf{q} \pm 2 \mathbf{Q})$ from Eq. (11) into Eq. (5) results in the following equation for $f_{0}$ :

$$
\begin{gathered}
{\left[L_{n}(\mathbf{q})+\frac{\tilde{t}^{2}}{L_{n}(\mathbf{q}+\mathbf{Q})}+\frac{\tilde{t}^{2}}{L_{n}(\mathbf{q}-\mathbf{Q})}\right] f_{0}} \\
-\frac{\tilde{t}^{2} \Delta_{ \pm 2}}{L_{n}(\mathbf{q} \pm \mathbf{Q}) L_{n}(\mathbf{q} \pm 2 \mathbf{Q})}=\Delta_{0} .
\end{gathered}
$$


Here we took into account that for $\mathbf{q}=-\mathbf{Q}, \Delta_{0} \sim \Delta_{2} \gg \Delta_{-2}$, while for $\mathbf{q}=\mathbf{Q}, \Delta_{0} \sim \Delta_{-2} \gg \Delta_{2}$. In the resonance $\mathbf{q}=\mathbf{Q}$ these equations acquire the form

$$
\begin{gathered}
{\left[L_{n}(-\mathbf{q})+\frac{\tilde{t}^{2}}{L_{n}(-2 \mathbf{q})}+\frac{\tilde{t}^{2}}{L_{n}(0)}\right] f_{ \pm 2}-\frac{\tilde{t}^{2} \Delta_{0}}{L_{n}(0) L_{n}(\mathbf{q})}=\Delta_{ \pm 2}} \\
{\left[L_{n}(\mathbf{q})+\frac{\tilde{t}^{2}}{L_{n}(2 \mathbf{q})}+\frac{\tilde{t}^{2}}{L_{n}(0)}\right] f_{0}-\frac{\tilde{t}^{2} \Delta_{ \pm 2}}{L_{n}(0) L_{n}(-\mathbf{q})}=\Delta_{0} .}
\end{gathered}
$$

Then Eq. (12) can be written as

$$
\begin{aligned}
& f_{0}=\Delta_{0}\left[\frac{1}{L_{n}(\mathbf{q})}-\frac{\tilde{t}^{2}}{L_{n}^{2}(\mathbf{q}) L_{n}(\mathbf{q}+\mathbf{Q})}-\frac{\tilde{t}^{2}}{L_{n}^{2}(\mathbf{q}) L_{n}(\mathbf{q}+\mathbf{Q})}\right] \\
& +\frac{\tilde{t}^{2} \Delta_{ \pm 2}}{L_{n}(\mathbf{q}) L_{n}(\mathbf{q} \pm \mathbf{Q}) L_{n}(\mathbf{q} \pm 2 \mathbf{Q})} \text {. } \\
& f_{ \pm 2}=\Delta_{ \pm 2}\left[\frac{1}{L_{n}(\mathbf{q} \pm 2 \mathbf{Q})}-\frac{\tilde{t}^{2}}{L_{n}^{2}(\mathbf{q} \pm 2 \mathbf{Q}) L_{n}(\mathbf{q} \pm 3 \mathbf{Q})}\right. \\
& \left.-\frac{\tilde{t}^{2}}{L_{n}^{2}(\mathbf{q} \pm 2 \mathbf{Q}) L_{n}(\mathbf{q} \pm \mathbf{Q})}\right] \\
& +\frac{\tilde{t}^{2} \Delta_{0}}{L_{n}(\mathbf{q}) L_{n}(\mathbf{q} \pm \mathbf{Q}) L_{n}(\mathbf{q} \pm 2 \mathbf{Q})}
\end{aligned}
$$

Making use of the self-consistency relation we obtain

$$
\begin{gathered}
\Delta_{0}\left[P+t^{2} a(\mathbf{q}, \mathbf{Q})\right]=\Delta_{ \pm 2} t^{2} c_{ \pm}(\mathbf{q}, \mathbf{Q}), \\
\Delta_{ \pm 2}\left[P+t^{2} a(\mathbf{q} \pm 2 \mathbf{Q}, \mathbf{Q})+\delta_{ \pm}\right]=\Delta_{0} t^{2} c_{ \pm}(\mathbf{q}, \mathbf{Q})
\end{gathered}
$$

where the following notations are introduced: $P=\left(T_{c}-\right.$ $\left.T_{c P}\right) / A T_{c}$ and $A=1-\frac{h}{T_{c} P} \frac{\partial T_{c P}}{\partial h}$. Here $T_{c P}$ is the superconducting onset temperature in the pure Pauli limit determined by

$$
\ln \left(\frac{T_{c 0}}{T_{c P}}\right)=\pi T_{c P} \sum_{n} \frac{1}{\omega_{n}}-\left\langle\frac{1}{L_{n}(\mathbf{q})}\right\rangle .
$$

The FFLO modulation vector $\mathbf{q}$ corresponds to the maximum of $T_{c P}(\mathbf{q}) . \Delta T_{c P}=T_{c}-T_{c P}$ is the shift of the critical temperature due to the orbital effect. In addition, the following notations are used:

$$
\begin{gathered}
\delta_{ \pm}(\mathbf{q}, \mathbf{Q})=\left.\pi T \sum_{n}\left\langle\frac{1}{L_{n}(\mathbf{q})}-\frac{1}{L_{n}(\mathbf{q} \pm 2 \mathbf{Q})}\right\rangle\right|_{T=T_{c} P}, \\
a(\mathbf{q}, \mathbf{Q})=\left.\pi T \sum_{n, \xi= \pm} T_{n}(\mathbf{q}, \mathbf{q}, \mathbf{q}+\xi \mathbf{Q})\right|_{T=T_{c P}}, \\
c_{ \pm}(\mathbf{q}, \mathbf{Q})=\left.\pi T \sum_{n} T_{n}(\mathbf{q}, \mathbf{q} \pm \mathbf{Q}, \mathbf{q} \pm 2 \mathbf{Q})\right|_{T=T_{c P}},
\end{gathered}
$$

where $T_{n}(\mathbf{q}, \mathbf{p}, \mathbf{k})=\left\langle L_{n}^{-1}(\mathbf{q}) L_{n}^{-1}(\mathbf{p}) L_{n}^{-1}(\mathbf{k})\right\rangle / 2$. In the IM model

$$
\left\langle\frac{1}{L_{n}(\mathbf{q})}\right\rangle=\left(\frac{|\mathbf{q}|^{2} v_{F}^{2}}{4}+\Omega_{n}^{2}\right)^{-1 / 2} .
$$

In the SM model the largest critical temperature corresponds to the wave vector $\mathbf{q}$ along the diagonals of the Fermi surface $q_{x}=q_{y}=q / \sqrt{2}$,

$$
\left\langle\frac{1}{L_{n}(\mathbf{q})}\right\rangle=\frac{1}{2} \sum_{ \pm} \frac{1}{\Omega_{n} \pm i v_{F} q_{x} / 2} .
$$

The solution of the system of Eqs. (17) and (18) is given as

$$
T_{c}=T_{c P}\left[1-A\left(S_{\mathrm{O}}+S_{\mathrm{R}}\right)\right]
$$

with the "orbital" term $S_{\mathrm{O}} \equiv t^{2} a(\mathbf{q}, \mathbf{Q})$ and the "resonance" term $S_{\mathrm{R}}=\min _{\xi} S_{\mathrm{R}}^{\xi}(\mathbf{q}, \mathbf{Q})$ with

$S_{\mathrm{R}}^{\xi}(\mathbf{q}, \mathbf{Q}) \equiv-\frac{\left(a-b_{\xi}\right) t^{2}-\delta_{\xi}}{2}-\frac{t^{2}}{2} \sqrt{\left[a-b_{\xi}-\delta_{\xi} / t^{2}\right]^{2}+4 c_{\xi}^{2}}$,

where $b_{\xi} \equiv a(\mathbf{q}+\xi 2 \mathbf{Q}, \mathbf{Q})$ and those values of $\xi= \pm$ are chosen that maximize the critical temperature. When the system is out of resonance, the second harmonic of the order parameter, $\Delta_{ \pm 2}$, can be neglected and the solution is just $T_{c}=T_{c P} /\left[1+A S_{\mathrm{O}}\right]$. However, if $\delta_{\xi}=0$ then the term $\left(P+t^{2} b+\delta_{\xi}\right)$ in the left-hand side of Eq. (18) is the same as the corresponding term in Eq. (17) and the precise resonance is established with $S_{\mathrm{R}}^{\xi}(\mathbf{q}, \mathbf{Q})=-c_{\xi} t^{2}$. In the IM model, the resonance occurs when $|\mathbf{q} \pm 2 \mathbf{Q}|=|\mathbf{q}|$, i.e., $\mathbf{q} \cdot \mathbf{Q}= \pm Q^{2}$. Due to the system isotropy the softening of the modes $\Delta_{0}$ and $\Delta_{ \pm 2}$ occurs simultaneously. When the role of the crystal anisotropy effects is important and there is only $\pm \mathbf{q}$ degeneracy for the FFLO vector, then the resonance occurs only for $\mathbf{Q}= \pm \mathbf{q}$. In the SM model the degeneracy of $\mathbf{q}$ is fourfold: $\pm \mathbf{q}_{1}$ and $\pm \mathbf{q}_{2}$ with $\mathbf{q}_{2} \perp \mathbf{q}_{1}$ and $\left|\mathbf{q}_{2}\right|=\left|\mathbf{q}_{1}\right|$ and $\mathbf{q}_{1}, \mathbf{q}_{2}$ are directed along the diagonals of the Fermi surface. So it is possible to have two resonance conditions: $\mathbf{Q}= \pm \mathbf{q}(|\mathbf{Q}|=|\mathbf{q}|)$ and $2 \mathbf{Q}=\mathbf{q}_{1} \pm \mathbf{q}_{2}(|\mathbf{Q}|=|\mathbf{q}| / \sqrt{2})$. The second resonance occurs at lower temperature.

\section{RESULTS AND DISCUSSION}

In our numerical investigations we restrict ourselves to the parameters of the compound $\kappa$-(BEDT-TTF $)_{2} \mathrm{Cu}(\mathrm{NCS})_{2}$. The Maki parameter $\alpha \simeq 8,{ }^{4}$ the interlayer coupling is $t=$ $1.5 \mathrm{~K},{ }^{33} t / T_{c 0}=0.16, \Delta_{0}=2.8 k T_{c 0},{ }^{34}$ and the Fermi velocity $v_{F}=5.0-10.0 \times 10^{4} \mathrm{~m} / \mathrm{ss}^{35}$ We have chosen the value $v_{F}=$ $7.5 \times 10^{4} \mathrm{~m} / \mathrm{s} .{ }^{33}$ Introducing the dimensionless Fermi velocity parameter $\eta=\hbar v_{F} \pi d / \phi_{0} \mu_{B}$, this value of $v_{F}$ corresponds to $\eta=2.55$. The summation over the Matsubara frequencies was performed numerically.

To illustrate the resonance condition between the magnetic wave vector $\mathbf{Q}=\left(-H_{y}, H_{x}\right) \pi d / \phi_{0}$ and the FFLO modulation wave vector $\mathbf{q}$, we display the amplitudes of these vectors in Figs. 1(a) and 1(b) as a function of reduced temperature $T_{c P} / T_{c 0}$ for the IM and SM models, respectively. The insets show the corresponding in-plane Fermi surfaces; the direction of the $\mathbf{q}$ vector is along the $x$ axis. At low temperature, the vectors $\mathbf{q}$ and $\mathbf{Q}$ exhibit saturation in the IM model, while they diverge in the SM model, since it has features of a 1D system. In the IM model [panel (a)], the intersection of $|\mathbf{q}|$ and $|\mathbf{Q}|$ curves occurs at a single value of $T_{c P} / T_{c 0}$ for $\eta<2.4$. For $2.4<\eta<2.7$ these curves intersect at two values of the reduced temperature, for instance for $\eta=2.55$ at $T_{c P} / T_{c 0}=$ 0.056 and $T_{c P} / T_{c 0}=0.32$, resulting in two resonances. For $\eta>2.7$ these curves do not intersect at all. In general, the 

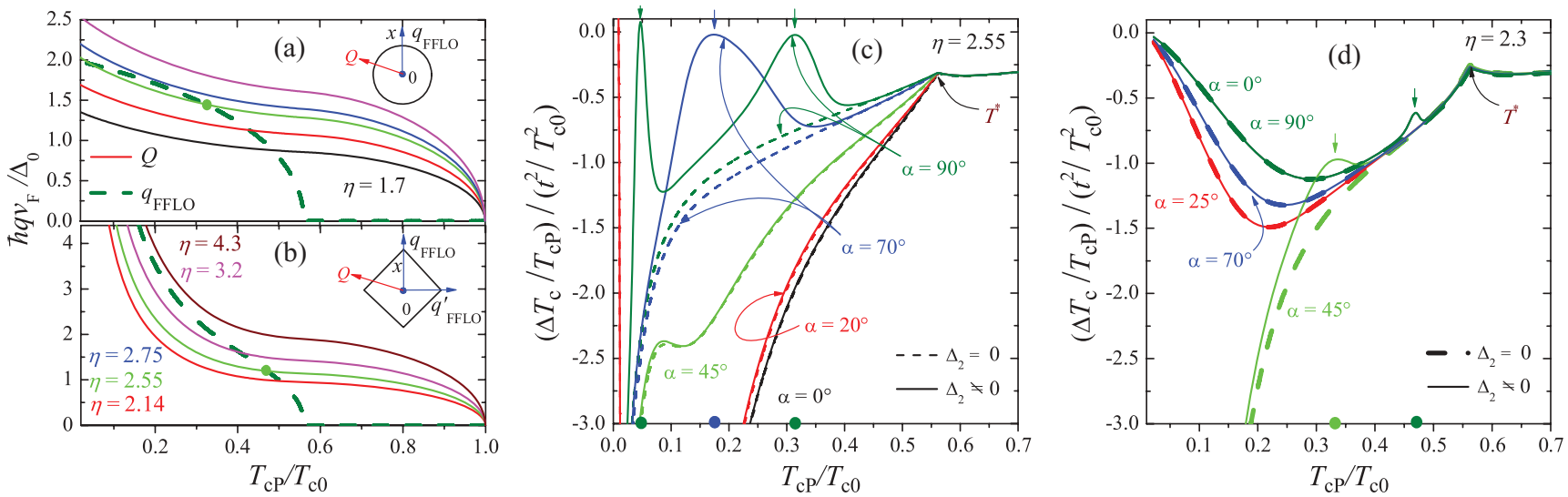

FIG. 1. (Color online) (a) Absolute value of the FFFLO wave vector $\mathbf{q}$ (dashed line) and of the magnetic wave vectors $\mathbf{Q}$ (solid lines) versus the reduced temperature $T_{c P} / T_{c 0}$ calculated for several values $\eta$ in the IM model (a) and in the SM model (b). (c), (d) Contribution of the orbital effect as a function of $T_{c P} / T_{c 0}$ for several angles $\alpha$ between $\mathbf{H}$ and $\mathbf{q}$. Solid lines are the results obtained for $\Delta_{2} \neq 0$; dashed lines are for $\Delta_{2}=0$ and they give an idea of the onset temperature variation far from resonance. The resonances are indicated by the straight arrows. (b) The IM model and (c) the SM model.

position of the resonance depends on $\alpha$ and, for instance in the IM model, for $\eta=2.55$, the resonance appears only in the range of angles $72^{\circ} \leqslant \alpha \leqslant 108^{\circ}$.

Figures 1(c) and 1(d) display the change of the transition temperature $\Delta T_{c}=T_{c}-T_{c P}$, due to the orbital effects of the applied magnetic field as a function of the reduced temperature for different field orientations, for the IM and SM models, respectively. The dashed lines illustrate the results of neglecting the second harmonics of the order parameter $\Delta_{ \pm 2}$, or the coupling between the equations in the system (17) and (18), while the solid lines are the solutions of the full system of these equations. One can see that two types of curves
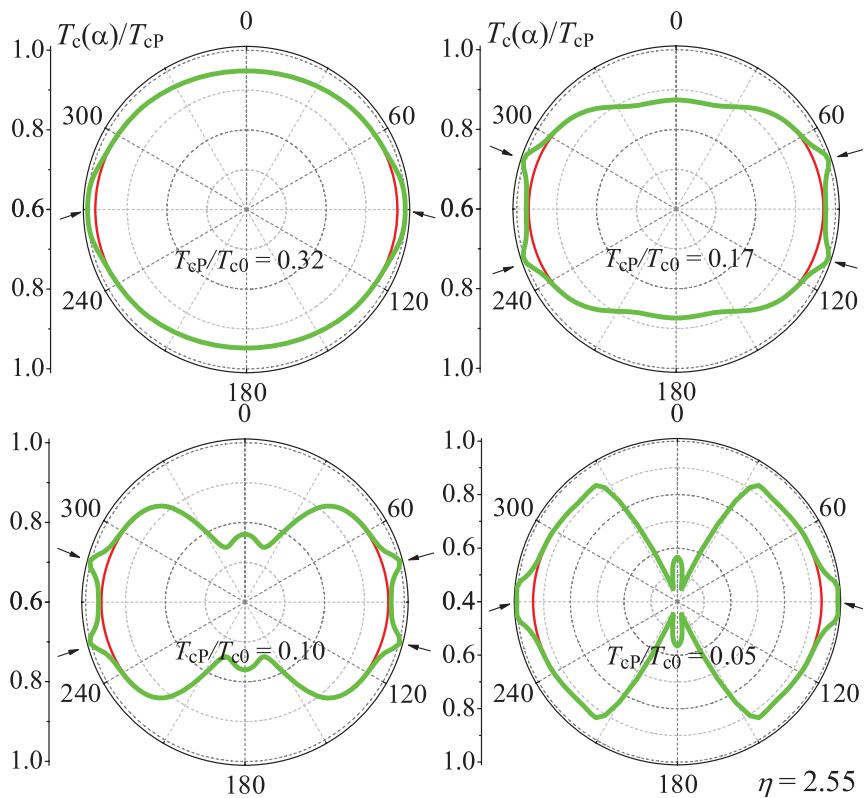

FIG. 2. (Color online) Normalized transition temperature $T_{c}(\alpha) / T_{c P}$ as a function of $\alpha$ for several values of $T_{c P} / T_{c 0}$ and for $t / T_{c 0}=0.16$ in the IM model. Thick lines are for $\Delta_{2} \neq 0$; thin lines are for $\Delta_{2}=0$. For the purpose of clarity the shown range of $T_{c}(\alpha) / T_{c P}$ is from 0.6 to 1.0 . almost coincide for angles $\alpha=0^{\circ}, 20^{\circ}, 45^{\circ}$ in the IM model and for angles $\alpha=25^{\circ}, 70^{\circ}$ in the SM model. However there are essential differences in the curve behavior for the angles $\alpha=70^{\circ}, 90^{\circ}$ in the IM model and for $\alpha=0^{\circ}, 45^{\circ}, 90^{\circ}$ in the SM model. These differences are induced by the resonance effect discussed above. For the sake of clarity we duplicate the resonance positions in Figs. 1(c) and 1(d) by thick dots on the $T$ axis. The curve $\Delta T_{c P}$ for $\alpha=70^{\circ}$ exhibits only one cusp at $T_{c P} / T_{c 0} \approx 0.2$. At $T_{c P} / T_{c 0} \approx 0.2, \delta_{\xi}$ is close to zero and this vicinity induces a wide pseudoresonance peak on the curve $\Delta T_{c P}$ for $\alpha=70^{\circ}$. Our results show that the resonance contribution $S_{\mathrm{R}}$ can become of the order of the orbital effect itself, $S_{\mathrm{O}}$. At exact resonances, indicated in Fig. 1(c) by straight

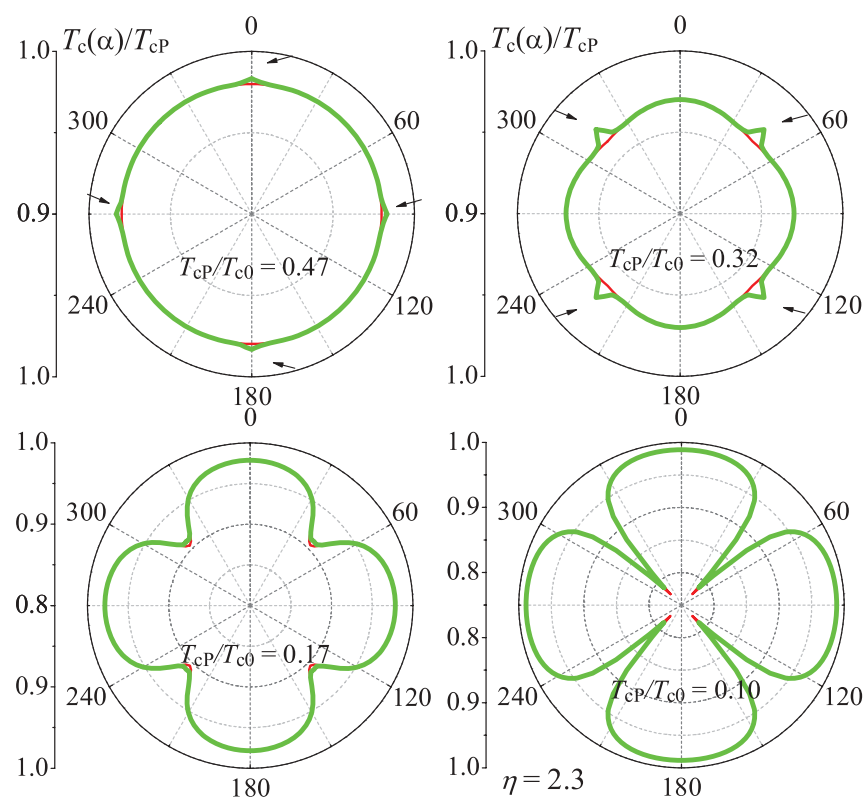

FIG. 3. (Color online) The same as in Fig. 2 but calculated within the SM model. The resonance at $T_{c P} / T_{c 0}=0.47$ occurs at $\mathbf{Q}= \pm \mathbf{q}_{1,2}$, while at $T_{c P} / T_{c 0}=0.32$ the resonance corresponds to $\mathbf{Q}= \pm\left(\mathbf{q}_{1} \pm\right.$ $\left.\mathbf{q}_{2}\right) / 2$. 

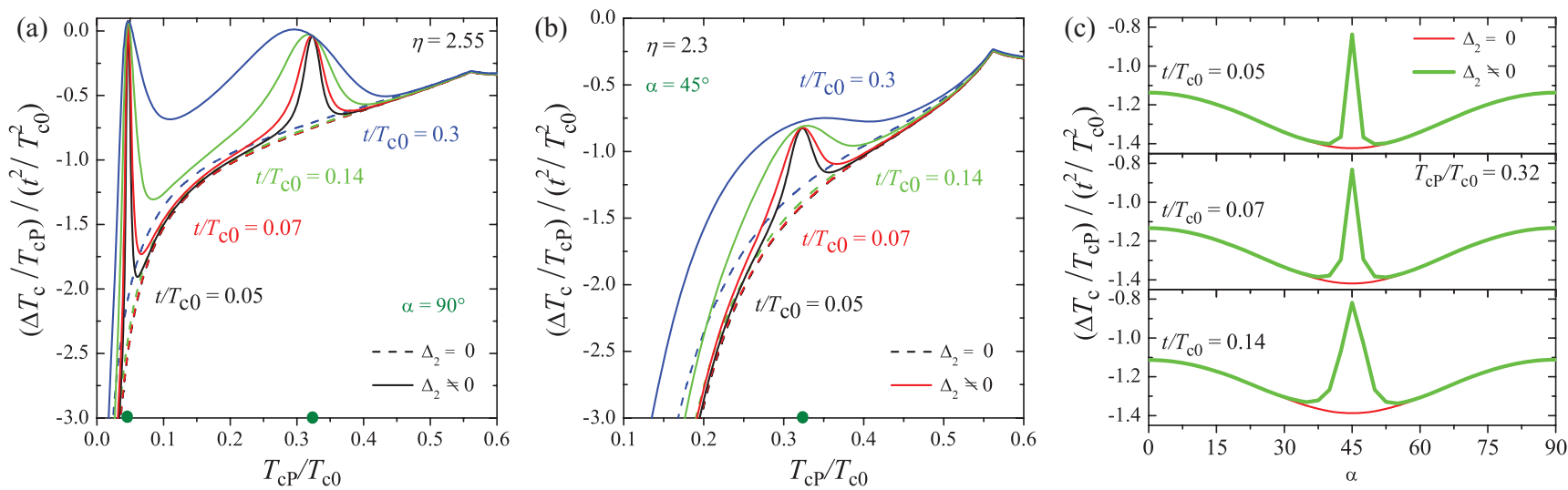

FIG. 4. (Color online) Contribution of the orbital effect as a function of $T_{c P} / T_{c 0}$ for several values of the parameter $t / T_{c 0}$ and for $\alpha=90^{\circ}$ (a) (IM model) and $\alpha=45^{\circ}$ (b) (SM model). Solid lines are the results obtained for $\Delta_{2} \neq 0$; dashed lines are for $\Delta_{2}=0$. (c) Normalized superconducting transition temperature $T_{c}(\alpha) / T_{c P}$ as a function of $\alpha$ for $\eta=2.3$ and $T_{c P} / T_{c 0}=0.32$ for several values of the parameter $t / T_{c 0}$ (SM model).

arrows, the suppression of the critical temperature due to the orbital effect becomes negligible.

The resonance effect results in particular features of the anisotropy of the superconducting onset temperature induced by the spatially modulated FFLO phase. Figure 2 shows the magnetic field angular dependence of the normalized superconducting transition temperature $T_{c}(\alpha) / T_{c P}$ calculated in the IM model at $T_{c P} / T_{c 0} \simeq 0.05,0.1,0.17$, and 0.32 , when accounting for the second harmonics of the order parameter $\Delta_{ \pm 2} \neq 0$ (solid line) and out of resonance $\Delta_{ \pm 2}=0$ (dashed line). In the polar plot the direction of each point seen from the origin corresponds to the magnetic field direction and the distance from the origin corresponds to the normalized critical temperature. We see that in addition to the overall anisotropy induced by the FFLO modulation and studied in Ref. 24, cusps develop for certain directions of the applied field, when the resonance conditions are realized. The cusp positions are marked by the straight arrows in Fig. 2. These cusps are the result of the resonant interplay between the FFLO wave vector and the magnetic wave vector, when the orbital effects of the field are taken in the second-order approximation. The incorporation in the model of the terms beyond the second order produces additional, although much smaller, peaks in the angular dependence of the upper critical field. Figure 3 illustrates the superconducting onset temperature as a function of the direction of the applied magnetic field calculated within the SM model at $T_{c P} / T_{c 0} \simeq 0.1,0.17,0.32$ and 0.47 . Due to the symmetry of the Fermi surface, $\mathbf{q}$ can be pinned in four directions: $0^{\circ}, \pm 90^{\circ}$, and $180^{\circ}$. This results in four cusps observed at $T_{c P} / T_{c 0} \simeq 0.32$ and 0.47 . At lower temperatures $T_{c P} / T_{c 0} \simeq$ 0.10 and 0.17 , the resonances disappear and the overall anisotropy is due to the FFLO modulation along $\mathbf{q}_{1}$ and $\mathbf{q}_{2}$.

Hitherto we restricted ourselves to the parameters of the organic compound $\kappa$-(ET) ${ }_{2} \mathrm{Cu}(\mathrm{NCS})_{2}$ with the interlayer coupling $t \simeq 1.5 \mathrm{~K}\left(t / T_{c 0} \simeq 0.16\right)$. In organic layered superconductor $\kappa$-(ET) $)_{2} \mathrm{Cu}(\mathrm{NCS})_{2} \mathrm{Br}, t \simeq 2.3 \mathrm{~K}$ and $t / T_{c 0} \simeq 0.19$. $^{36}$ The developed theory is quantitatively valid for any layered superconducting materials as soon as $t / T_{c 0} \ll 1$ and we expect that it gives qualitatively correct results for the anisotropy of the upper critical field even if $t / T_{c 0} \lesssim 1$. Therefore it may be interesting to study how the anisotropy parameter $t / T_{c 0}$ influences $H_{c 2}^{\|}$in the close vicinity of resonances. With this aim in mind we consider two of our models (IM and SM), and calculate a change of the transition temperature $\Delta T_{c P}$ due to the applied field-induced orbital effects as a function of the reduced temperature for $\mathbf{Q} \| \mathbf{q}$ and $\mathbf{Q} \|\left(\mathbf{q}_{1}-\mathbf{q}_{2}\right) / 2$, respectively. The results are displayed in Figs. 4(a) and 4(b). Figure 4(c) illustrates the angular dependence of the normalized $\Delta T_{C P}$ versus the applied field direction. One can see that the height of the resonance peak normalized on the parameter $\left(t / T_{c 0}\right)^{2}$ remains unaltered by the interlayer coupling strength. However the width of the peak becomes narrower with decreasing $t / T_{c 0}$.

The relative shift of the critical temperature due to the orbital effect is proportional to $\left(t / T_{c 0}\right)^{2}$. So for the experimental observation of the proposed resonance effects it is better to choose the layered compound with not too small anisotropy parameter $t / T_{c 0}$ and to first study the overall angular dependence of the critical field in the FFLO phase. This should permit one to determine the direction of the FFLO modulation ${ }^{24}$ and then orienting the applied field perpendicular to this direction would create the optimal conditions for the resonance effect observation.

\section{CONCLUSIONS}

In conclusion, we have shown that in layered superconductors under the applied in-plane magnetic field in the FFLO phase the resonance between the modulation wave vector and the vector potential may lead to anomalous cusps in the field-direction dependence of the upper critical field. Therefore, we suggest that observation of characteristic cusps in the anisotropy of the onset of superconductivity may be direct evidence for the appearance of the FFLO phase in layered superconductors.

\section{ACKNOWLEDGMENTS}

We acknowledge the support by the European Community under a Marie Curie IEF Action (Grant Agreement No. PIEF-GA-2009-235486-ScQSR), the French Project "SINUS" ANR-09-BLAN-0146. We are grateful to M. Houzet for useful comments and discussions. 
${ }^{1}$ A. I. Buzdin and L. N. Bulaevskii, Sov. Phys. Usp. 27, 830 (1984).

${ }^{2}$ The Physics of Organic Superconductors and Conductors, edited by A. G. Lebed (Springer, Berlin, 2008).

${ }^{3}$ R. Lortz, Y. Wang, A. Demuer, P. H. M. Bottger, B. Bergk, G. Zwicknagl, Y. Nakazawa, and J. Wosnitza, Phys. Rev. Lett. 99, 187002 (2007).

${ }^{4}$ B. Bergk, A. Demuer, I. Sheikin, Y. Wang, J. Wosnitza, Y. Nakazawa, and R. Lortz, Phys. Rev. B 83, 064506 (2011).

${ }^{5}$ S. Yonezawa, S. Kusaba, Y. Maeno, P. Auban-Senzier, C. Pasquier, K. Bechgaard, and D. Jerome, Phys. Rev. Lett. 100, 117002 (2008).

${ }^{6}$ A. I. Larkin and Yu. N. Ovchinnikov, Zh. Eksp. Teor. Phys. 47, 1136 (1964) [Sov. Phys. JETP 20, 762 (1965)].

${ }^{7}$ P. Fulde and R. A. Ferrell, Phys. Rev. 135, A550 (1964).

${ }^{8}$ S. Yonezawa, S. Kusaba, Y. Maeno, P. Auban-Senzier, C. Pasquier, and D. Jérome, J. Phys. Soc. Jpn. 77, 054712 (2008).

${ }^{9}$ L. G. Aslamazov, Zh. Eksp. Teor. Phys. 55, 1477 (1968) [Sov. Phys. JETP 28, 773 (1969)].

${ }^{10}$ M. Houzet and V. P. Mineev, Phys. Rev. B 74, 144522 (2006).

${ }^{11}$ Y. Matsuda and H. Shimahara, J. Phys. Soc. Jpn. 76, 051005 (2007).

${ }^{12}$ M.-S. Nam, J. A. Symington, J. Singleton, S. J. Blundell, A. Ardavan, J. A. A. J. Perenboom, M. Kurmoo, and P. Day, J. Phys.: Condens. Matter 11, L477 (1999).

${ }^{13}$ J. Singleton, J. A. Symington, M.-S. Nam, A. Ardavan, M. Kurmoo, and P. Day, J. Phys.: Condens. Matter 12, L641 (2000).

${ }^{14}$ J. A. Wright, E. Green, P. Kuhns, A. Reyes, J. Brooks, J. Schlueter, R. Kato, H. Yamamoto, M. Kobayashi, and S. E. Brown, Phys. Rev. Lett. 107, 087002 (2011).

${ }^{15}$ M. A. Tanatar, T. Ishiguro, H. Tanaka, and H. Kobayashi, Phys. Rev. B 66, 134503 (2002).

${ }^{16}$ R. Modler, P. Gegenwart, M. Lang, M. Deppe, M. Weiden, T. Lühmann, C. Geibel, F. Steglich, C. Paulsen, J. L. Tholence, N. Sato, T. Komatsubara, Y. Ōnuki, M. Tachiki, and S. Takahashi, Phys. Rev. Lett. 76, 1292 (1996).

${ }^{17}$ A. Yamashita, K. Ishii, T. Yokoo, J. Akimitsu, M. Hedo, Y. Inada, Y. Onuki, E. Yamamoto, Y. Haga, and R. Kadono, Phys. Rev. Lett. 79, 3771 (1997).
${ }^{18}$ A. Bianchi, R. Movshovich, N. Oeschler, P. Gegenwart, F. Steglich, J. D. Thompson, P. G. Pagliuso, and J. L. Sarrao, Phys. Rev. Lett. 89, 137002 (2002).

${ }^{19}$ C. F. Miclea, M. Nicklas, D. Parker, K. Maki, J. L. Sarrao, J. D. Thompson, G. Sparn, and F. Steglich, Phys. Rev. Lett. 96, 117001 (2006).

${ }^{20}$ L. N. Bulaevskii, Zh. Eksp. Teor. Fiz. 65, 1278 (1973) [Sov. Phys. JETP 38, 634 (1974)].

${ }^{21}$ A. Buzdin and J. P. Brison, Europhys. Lett. 35, 707 (1996).

${ }^{22}$ M. Houzet and A. Buzdin, Europhys. Lett. 50, 375 (2000).

${ }^{23}$ H. Shimahara, Phys. Rev. B 80, 214512 (2009).

${ }^{24}$ M. D. Croitoru, M. Houzet, and A. I. Buzdin, Phys. Rev. Lett. 108, 207005 (2012); J. Supercond. Novel Magn. 25, 1283 (2012).

${ }^{25}$ M. Yoshimura, H. Shigekawa, H. Nejoh, G. Saito, Y. Saito, and A. Kawazu, Phys. Rev. B 43, 13590 (1991).

${ }^{26}$ D. Saint-James, G. Sarma, and E. J. Thomas, Type II Superconductivity (Pergamon, Oxford, 1969).

${ }^{27}$ L. Bulaevskii, A. Buzdin, and M. Maley, Phys. Rev. Lett. 90, 067003 (2003).

${ }^{28}$ S. Uji, T. Terashima, M. Nishimura, Y. Takahide, T. Konoike, K. Enomoto, H. Cui, H. Kobayashi, A. Kobayashi, H. Tanaka, M. Tokumoto, E. S. Choi, T. Tokumoto, D. Graf, and J. S. Brooks, Phys. Rev. Lett. 97, 157001 (2006).

${ }^{29}$ J. P. Brison, N. Keller, A. Vernière, P. Lejay, L. Schmidt, A. Buzdin, J. Flouquet, S. R. Julian, and G. G. Lonzarich, Physica C 250, 128 (1995).

${ }^{30}$ T. Tsuzuki, J. Low. Temp. Phys. 9, 525 (1972).

${ }^{31}$ N. B. Kopnin, Theory of Nonequilibrium Superconductivity (Clarendon, Oxford, 2001).

${ }^{32}$ D. Denisov, A. Buzdin, and H. Shimahara, Phys. Rev. B 79, 064506 (2009).

${ }^{33}$ J. Singleton, P. A. Goddard, A. Ardavan, N. Harrison, S. J. Blundell, J. A. Schlueter, and A. M. Kini, Phys. Rev. Lett. 88, 037001 (2002).

${ }^{34}$ J. Müller, M. Lang, R. Helfrich, F. Steglich, and T. Sasaki, Phys. Rev. B 65, 140509 (2002).

${ }^{35}$ K. Izawa, H. Yamaguchi, T. Sasaki, and Y. Matsuda, Phys. Rev. Lett. 88, 027002 (2001).

${ }^{36}$ Á. Antal, T. Feher, A. Janossy, E. Tatrai-Szekeres, and F. Fulop, Phys. Rev. Lett. 102, 086404 (2009). 